\title{
DECOMPOSABILITY OF FINITE RINGS
}

\author{
DLANE MAINWARING and K. R. PEARSON
}

(Received 18 August 1978; revised 16 February 1979)

Communicated by R. Lidl

\begin{abstract}
Let $p$ be a prime, let $R$ be a finite $p$-ring with identity and suppose that the radical of $R$ has $p^{m}$ elements. If $R$ is indecomposable as a ring then there are at most $m+1$ minimal ideals in $R / \operatorname{Rad} R$. 1980 Mathematics subject classification (Amer. Math. Soc.): 16 A 44.
\end{abstract}

Keywords: Finite ring, decomposable, nilpotent p-ring, group of units.

Our main result is the following theorem.

THEOREM. Let $p$ be a prime, let $R$ be a finite p-ring with identity and suppose $\operatorname{Rad} R$ has $p^{m}$ elements. If $R$ is indecomposable as a ring then there are at most $m+1$ minimal ideals in $R / \operatorname{Rad} R$.

Stewart (1972), Theorem 3.3, has proved a related result for the case $p=2$ and the above theorem improves his result, obtained via Lemma 3.4, that $\mu(J) \leqslant 2 \mathrm{~m}^{2}$ to $\mu(J) \leqslant m+1$. Other results bounding the size of rings with a given radical are Theorem 3.8 of Hall (1940) and Theorem 6.2 of Flanigan (1973).

We also prove the following result which improves the bound given in Theorem 3.5 of Stewart (1972).

Proposition. Let $R$ be a finite indecomposable 2-ring with identity and with group of units $G$. If $R / \operatorname{Rad} R$ has $t$ simple summands distinct from $\mathrm{Z}_{2}$ then

$$
|R| \leqslant 2^{1-t}|G|^{2} \text {. }
$$

The results of this paper appear in Mainwaring (1978) and are one of the major tools used there to describe completely all finite rings whose unit groups are dihedral. 
ProOf OF THE THEOREM. Suppose that $R$ is indecomposable and that

$$
\bar{R}=R / \operatorname{Rad} R=Y_{1} \oplus \ldots \oplus Y_{s}
$$

where each $Y_{i}$ is a matrix ring over a finite field of characteristic $p$ : we must prove that $s \leqslant m+1$. It is well known (see, for example, Proposition 5, page 54 of Jacobson (1964)) that there are orthogonal idempotents $f_{1}, \ldots, f_{s}$ in $R$ whose sum is 1 such that $v\left(f_{i}\right)=\delta_{i}$ where $\delta_{1}, \ldots, \delta_{s}$ are the obvious $s$ central orthogonal idempotents in $\bar{R}$ whose sum is 1 and $v: R \rightarrow \bar{R}$ is the natural map. Notice that $R$ is an internal direct sum (as an abelian group) of the $f_{i} R f_{j}(1 \leqslant i, j \leqslant s)$ and that if $i \neq j$,

$$
v\left(f_{i} R f_{j}\right)=\delta_{i} \bar{R} \delta_{j}=0
$$

so that $f_{i} R f_{j} \subseteq \operatorname{Rad} R$. Thus

$$
\prod_{i \neq j}\left|f_{i} R f_{j}\right| \leqslant|\operatorname{Rad} R|=p^{m}
$$

which means that the number $k$ of nonzero $f_{i} R f_{j}$ with $i \neq j$ is at most $m$.

Notice that if $A_{1}$ and $A_{2}$ are nonempty disjoint sets whose union is $\{1,2, \ldots, s\}$ then there exist $i \in A_{1}$ and $j \in A_{2}$ such that either $f_{i} R f_{j} \neq 0$ or $f_{j} R f_{i} \neq 0$, since otherwise $R=R_{1} \oplus R_{2}$ where each

$$
R_{l}=\sum_{i, j \in A_{i}} f_{i} R f_{j}
$$

is an ideal of $R$ since the $f$ 's are orthogonal. If we take $A_{1}$ successively as $\{1\},\left\{1, i_{1}\right\}, \ldots,\left\{1, i_{1}, \ldots, i_{s-2}\right\}$ we see that there exist $i_{1}, \ldots, i_{s-1}$ all distinct from 1 and $j_{1}, \ldots, j_{s-2}$ with $j_{l} \in\left\{1, i_{1}, \ldots, i_{l}\right\}$ such that each of

$$
f_{1} R f_{i_{1}} \cup f_{i_{1}} R f_{1}, \quad f_{j_{1}} R f_{i_{2}} \cup f_{i_{2}} R f_{j_{1}}, \quad \ldots, \quad f_{j_{s-2}} R f_{i_{s-1}} \cup f_{i_{s-1}} R f_{j_{s-2}}
$$

is nonzero. This leads to at least $s-1$ different nonzero $f_{\mathrm{i}} R f_{j}$ with $i \neq j$ and hence $s-1 \leqslant k \leqslant m$, as required.

ProOf OF THE Proposition. Let $J=\operatorname{Rad} R$, put $|J|=2^{m}$ and let $R / J \simeq Y \oplus w Z_{2}$ where $Y$ is a direct sum of $t$ matrix rings over fields of characteristic 2 and includes no $Z_{2}$ summands. By the theorem above, $t+w \leqslant m+1$. We denote by $W^{*}$ the group of units of any ring $W$. Since $R^{*} /(1+J) \simeq Y^{*}$ (McDonald (1974), Theorem XX1.5) and since $|Y| \leqslant\left|Y^{*}\right|^{2}$ by Lemma 2.3 of Stewart (1972),

$$
|R|=|J||Y| 2^{w} \leqslant|J|^{2}\left|Y^{*}\right|^{2} 2^{w-m}=\left|R^{*}\right|^{2} 2^{w-m} \leqslant 2^{1-t}|G|^{2} .
$$

\section{References}

F. J. Flanigan (1973), 'Radical behaviour and the Wedderburn family', Bull. Amer. Math. Soc., 79, 66-70.

F. J. Flanigan (1974), 'Radical embedding, genus and toroidal derivations of nilpotent associative algebras', Bull. Amer. Math. Soc. 80, 986-990. 
Marshall Hall (1940), 'The position of the radical in an algebra', Trans. Amer. Math. Soc. 48, $391-404$.

Nathan Jacobson (1964), Structure of rings, 2nd ed. (Amer. Math. Soc. Colloq. Publ., Providence). Diane Mainwaring (1978), 'Finite rings with dihedral groups of units', M.Sc. thesis, La Trobe University.

Bernard R. McDonald (1974), Finite rings with identity (Marcel Dekker, New York).

Ian Stewart (1972), 'Finite rings with a specified group of units', Math. Z. 126, 51-58 (and 128, 187).

La Trobe University

Bundoora, Victoria 3083

Australia 